\title{
Expression of receptor protein tyrosine phosphatase $\zeta$ is a risk factor for triple negative breast cancer relapse
}

\author{
FENFEN FU, XI XIAO, TAO ZHANG, QIONGYAN ZOU, ZONGLIN CHEN, LEI PEI, JUAN SU and WENJUN YI
}

\author{
Department of Breast and Thyroid Surgery, The Second Xiangya Hospital, \\ Central South University, Changsha, Hunan 410011, P.R. China
}

Received June 12, 2015; Accepted December 31, 2015

DOI: $10.3892 /$ br.2016.570

\begin{abstract}
Patients with triple negative breast cancer (TNBC) have a higher rate of distant recurrence and a poorer prognosis than those with other breast cancer subtypes. Therefore, it is important to study the mechanism of TNBC relapse. A retrospective immunohistochemical analysis of the expression of receptor protein tyrosine phosphatase $\zeta$ (PTPRZ1) and pleiotrophin (PTN) was performed for 325 cases of breast cancer. These samples included 66 cases of luminal A breast cancer, 67 cases of luminal B breast cancer, 78 cases of Her-2-enriched breast cancer, 78 cases of TNBC and 36 cases of relapsed TNBC (RTNBC). In addition, 30 control specimens and 30 cases of metastasized lymph nodes were examined. PTPRZ1 and PTN were highly expressed in the RTNBC group. Compared with the RTNBC group, significant differences in the expression of PTPRZ1 were observed between the TNBC, BC and control groups. A significant difference was observed in the expression of PTN in the BC group $(\mathrm{P}<0.05)$ compared to RTNBC, and there were no significant differences in the expression of PTPRZ1 and PTN among the molecular subtypes. No significant correlation was observed between the expression of PTPRZ1, PTN, ER, PR, Her-2 and ALN and the tumor size or menopause status. No significant correlation was identified between the expression of PTPRZ1 and PTN and the expression of CD24 and CD44. In summary, high expression of PTPRZ1 may be an independent risk indicator for TNBC recurrence and metastasis.
\end{abstract}

Correspondence to: Dr Wenjun Yi, Department of Breast and Thyroid Surgery, The Second Xiangya Hospital, Central South University, 139 Middle Renmin Road, Changsha, Hunan 410011, P.R. China

E-mail: alvinyi@yeah.net

Key words: breast cancer, triple negative breast cancer, relapse, protein tyrosine phosphatase $\zeta$, pleiotrophin

\section{Introduction}

Breast cancers (BCs) that are defined by a lack of demonstrable expression of the estrogen and progesterone receptors (ER and PR), and human epidermal growth factor receptor 2 (HER2) protein represent $\sim 15-20 \%$ of all breast cancers and have been labeled 'triple-negative' breast cancers (TNBCs). TNBCs affect younger patients more frequently, and have an increased prevalence in African-American women. TNBC tumors are typically larger in size, of higher grade, exhibit lymph node involvement at diagnosis, and are more biologically aggressive than other breast cancer tumors $(1,2)$.

Currently, there are limited therapeutic options available for TNBC, and apart from surgery, conventional chemotherapy is likely the only effective treatment for TNBC patients. Despite having higher rates of clinical response to neoadjuvant chemotherapy, TNBC patients also have a higher rate of distant recurrence and a poorer prognosis than patients with other breast cancer subtypes. Fewer than $30 \%$ of women with metastatic TNBC survive for 5 years, and almost all die from their disease, despite adjuvant chemotherapy $(2,3)$. Furthermore, several previous studies have demonstrated that TNBC is more sensitive to adjuvant or neoadjuvant chemotherapy than other subtypes of $\mathrm{BC}(1,4)$. Previous neoadjuvant trials have also shown that TNBC is correlated with better prognosis. Within the TNBC group, those who attained a pathological complete response (pCR) had an overall survival similar to that of patients in the non-TNBC group who also attained a pCR. However, in comparison with the patients who did not reach pCR in both groups, those with TNBC experienced a worse outcome $(5,6)$. The risk of recurrence was higher in the TNBC group during the first three years but not thereafter $(7,8)$; moreover, the median survival time from recurrence to death was significantly shorter in patients with the TNBC subtype compared with patients in the non-TNBC group (4). Unfortunately, only a minority of patients with TNBC are extremely sensitive to chemotherapy, with a good potential outcome. However, at present the reasons for the majority of TNBC patients being insensitive to chemotherapy remain unknown.

Receptor protein tyrosine phosphatase $\zeta$ (PTPRZ1), a member of the PTPR family, is a single-pass type I membrane protein with two cytoplasmic tyrosine phosphatase domains (D1 and D2), an $\alpha$ carbonic anhydrase domain (CA), 
chondroitin sulfate proteoglycans (CS-PGs) and a fibronectin type-III domain (FNIII) (9). PTPRZ1 interacts with its ligand pleiotrophin (PTN), a secreted growth factor that is involved in angiogenesis and tumor growth $(10,11)$. Upon binding, PTN inactivates the phosphatase activity of PTPRZ1, which leads to an increased phosphorylation of tyrosine residues in important signaling molecules such as $\beta$-catenin, Fyn and the RhoGAP domain (12-16).

Rokavec et al (16) identified that PTPRZ1 may be involved in the initiation and maintenance of breast and prostate cancer. The expression of miR-200c in both human breast and prostate cancer cells is inversely correlated with the expression level of interleukin-6 (IL-6), nuclear factor- $\kappa \mathrm{B}(\mathrm{NF}-\kappa \mathrm{B})$ activity, and phosphorylation of JNK2. Silencing of IL-6 induces miR-200c expression, whereas the overexpression of miR-200c represses IL-6 expression in MDA-MB-231 cells. The IL-6-miR-200c-JNK2-p65 circuit is present in human cancer cells. miR-200c downregulates PTPRZ1, which contributes to the constitutive phosphorylation of I $\kappa \mathrm{B} \alpha$, and this downregulation results in the constitutive activation of p65. Furthermore, ER $\alpha$ may be involved in the IL-6-induced downregulation of miR-200c. Therefore, PTPRZ1 may be important in chemoresistance and relapse in cases of TNBC.

\section{Materials and methods}

Human cancer samples. Samples were obtained with informed consent from each individual patient. The present study was approved by the Ethics Committee of the Second Xiangya Hospital of Central South University, Changsha, China. Between January 2010 and December 2013, a total of 1852 patients with breast cancer were treated at the Second Xiangya Hospital, Changsha, China. All primary cancers with a pathologic diagnosis based on the classification scheme of the WHO classification were selected for review (17). The following cases were selected for inclusion in the present study: 66 cases with the luminal A molecular subtype (A), 67 cases with the luminal B molecular subtype (B), 78 cases with the Her-2-enriched molecular subtype $(\mathrm{H}), 78$ cases with the TNBC molecular subtype (T), and 36 cases with TNBC that relapsed within the first three years (RTNBC). Thirty cases of breast fibroadenoma were included as the control group $(\mathrm{CON})$.

Antibodies. The anti-human antibodies that were used in the present study include: Anti-PTPRZ1 (cat no. 55125) from Sigma-Aldrich, St. Louis, MO, USA, and anti-pleiotrophin (cat no. 10821), anti-CD24 (cat no. 18330), and anti-CD44 (cat no. 60224) from Millipore, Billerica, MA, USA.

Immunohistochemistry (IHC). All IHC analyses were performed on paraffin-embedded tissues that were obtained from the primary tumor surgical specimen. For all IHC analyses, the surgically resected specimens were fixed in $10 \%$ formalin and embedded in paraffin for routine pathological examination. 5- $\mu$ m-thick paraffin sections were cut from a paraffin block and we ensured that each section contained histological findings that were representative of the tumor. Antigen retrieval was performed using citrate buffer solution ( $\mathrm{pH}$ 6.0).
Endogenous peroxidase was blocked with $0.3 \% \mathrm{H}_{2} \mathrm{O}_{2}$ in methanol for $15 \mathrm{~min}$, and all slides were microwaved to $95^{\circ} \mathrm{C}$ for $20 \mathrm{~min}$ and then cooled at room temperature (RT). The slides were then washed in phosphate-buffered saline, and after a 1-h incubation at RT with the primary antibodies, the slides were incubated for 30 min with Polymer EnVision $\mathrm{TM}^{+}$(peroxidase-conjugated anti-mouse or anti-rabbit; Dako, Tokyo, Japan). The chromogen was $2 \%$ 3,3'-diaminobenzidine in $50 \mathrm{mM}$ Tris-buffer ( $\mathrm{pH}$ 7.6) containing $0.3 \% \mathrm{H}_{2} \mathrm{O}_{2}$.

Statistical methods. Data processing and statistical analyses were conducted using the SPSS version 19.0 software package. The data were analyzed through a one-sample $t$ test, one-way ANOVA test or Pearson's correlation test. $\mathrm{P}<0.05$ was considered to represent a statistically significant difference.

\section{Results}

PTPRZ1 is highly expressed in the RTNBC group of patients. The immunohistochemical evaluations of 325 cases of breast cancer samples were analyzed in terms of PTPRZ1 and PTN expression. The samples included 66 cases of luminal A breast cancer, 67 cases of luminal B breast cancer, 78 cases of Her-2-enriched cancer, 78 cases of TNBC and 36 cases of RTNBC. Additionally, 30 control specimens and 30 cases of metastasized lymph nodes (LNs) were examined. Cytoplasmic yellow or tan staining was considered positive (Fig. 1). PTPRZ1 and PTN were both highly expressed in patients in the RTNBC group compared with patients in the RTNBC group. Significant differences were observed in the expression of PTPRZ1 among the TNBC, BC and control groups, and a significantly different expression of PTN was also observed in the $\mathrm{BC}$ group $(\mathrm{P}<0.05)$. However, no significant difference was noted in the comparisons between the other groups (Table I and Fig. 2).

Expression of PTPRZ1 and PTN in each molecular subtype of breast cancer. No significant differences were identified in the expression of PTPRZ1 and PTN among the molecular subtypes (A, B, H and T) (Table II and Fig. 3).

Association among PTPRZI, PTN and the clinical pathological features of patients with breast cancer. No significant correlation was observed among the expression of PTPRZ1, PTN, ER, PR, Her-2 and ALN and the tumor size and whether the patient had begun menopause $(\mathrm{P}>0.05)$ (Table III).

\section{Correlation between the expression of PTPRZ1 and PTN and} the expression of $C D 24$ and $C D 44$. To study the correlation between PTPRZ1 and PTN and the expression of CD24 and CD44, the expression of CD24 and CD44 was analyzed in 325 cases of breast cancer by IHC. No significant correlations between the expression of PTPRZ1 and PTN and the expression of CD24 and CD44 were found ( $P>0.05$ ) (Table IV).

\section{Discussion}

TNBCs are not a truly homogeneous group with biologically uniform behaviors. Classic morphological analyses using light microscopy and simple IHC have led to the identification 
Table I. Differential expression of PTPRZ1 and PTN in the RTNBC, TNBC, BC, LN and control groups.

\begin{tabular}{|c|c|c|c|c|c|}
\hline \multirow[b]{2}{*}{ Group } & \multirow[b]{2}{*}{ No. } & \multicolumn{2}{|c|}{ PTN } & \multicolumn{2}{|c|}{ PTPRZ1 } \\
\hline & & Mean \pm SD & P-value & Mean \pm SD & P-value \\
\hline RTNBC & 36 & $277.32 \pm 191.75$ & 0.050 & $760.21 \pm 494.87$ & 0.129 \\
\hline TNBC & 78 & $247.76 \pm 232.45$ & & $345.10 \pm 240.83^{b}$ & \\
\hline $\mathrm{BC}$ & 211 & $140.01 \pm 84.14^{\mathrm{a}}$ & & $395.20 \pm 370.58^{c}$ & \\
\hline $\mathrm{LN}$ & 30 & $122.03 \pm 45.75$ & & $353.77 \pm 89.69$ & \\
\hline Control & 30 & $135.79 \pm 121.44$ & & $336.62 \pm 89.39^{d}$ & \\
\hline
\end{tabular}

Compared with the RTNBC group, ${ }^{\mathrm{a}} \mathrm{P}=0.029$; compared with the RTNBC group, ${ }^{\mathrm{b}} \mathrm{P}=0.015,{ }^{\mathrm{c}} \mathrm{P}=0.017,{ }^{\mathrm{d}} \mathrm{P}=0.042$. BC group: luminal A, luminal B and Her-2 enriched group. PTPRZ1, protein tyrosine phosphatase $\xi ;$ PTN, pleiotrophin; TNBC, triple negative breast cancer; RTNBC, relapsed TNBC; LN, lymph node; CON, control group; SD, standard deviation.

Table II. Differential expression of PTN and PTPRZ1 in molecular subtypes of breast cancer.

\begin{tabular}{|c|c|c|c|c|c|}
\hline \multirow[b]{2}{*}{ Subtype } & \multirow[b]{2}{*}{ No. } & \multicolumn{2}{|c|}{ PTN } & \multicolumn{2}{|c|}{ PTPRZ1 } \\
\hline & & Mean \pm SD & P-value & Mean \pm SD & P-value \\
\hline A & 66 & $153.09 \pm 93.71$ & 0.055 & $334.63 \pm 232.43$ & 0.809 \\
\hline B & 67 & $122.53 \pm 109.64$ & & $419.42 \pm 495.78$ & \\
\hline $\mathrm{H}$ & 78 & $143.72 \pm 47.66$ & & $425.94 \pm 366.50$ & \\
\hline $\mathrm{T}$ & 114 & $257.09 \pm 215.49$ & & $476.19 \pm 382.09$ & \\
\hline
\end{tabular}

A, Luminal A molecular subtype; B, luminal B molecular subtype; H, Her-2-enriched molecular subtype; T, TNBC molecular subtype and RTNBC molecular subtype. PTPRZ1, protein tyrosine phosphatase $\zeta$; PTN, pleiotrophin; SD, standard deviation.
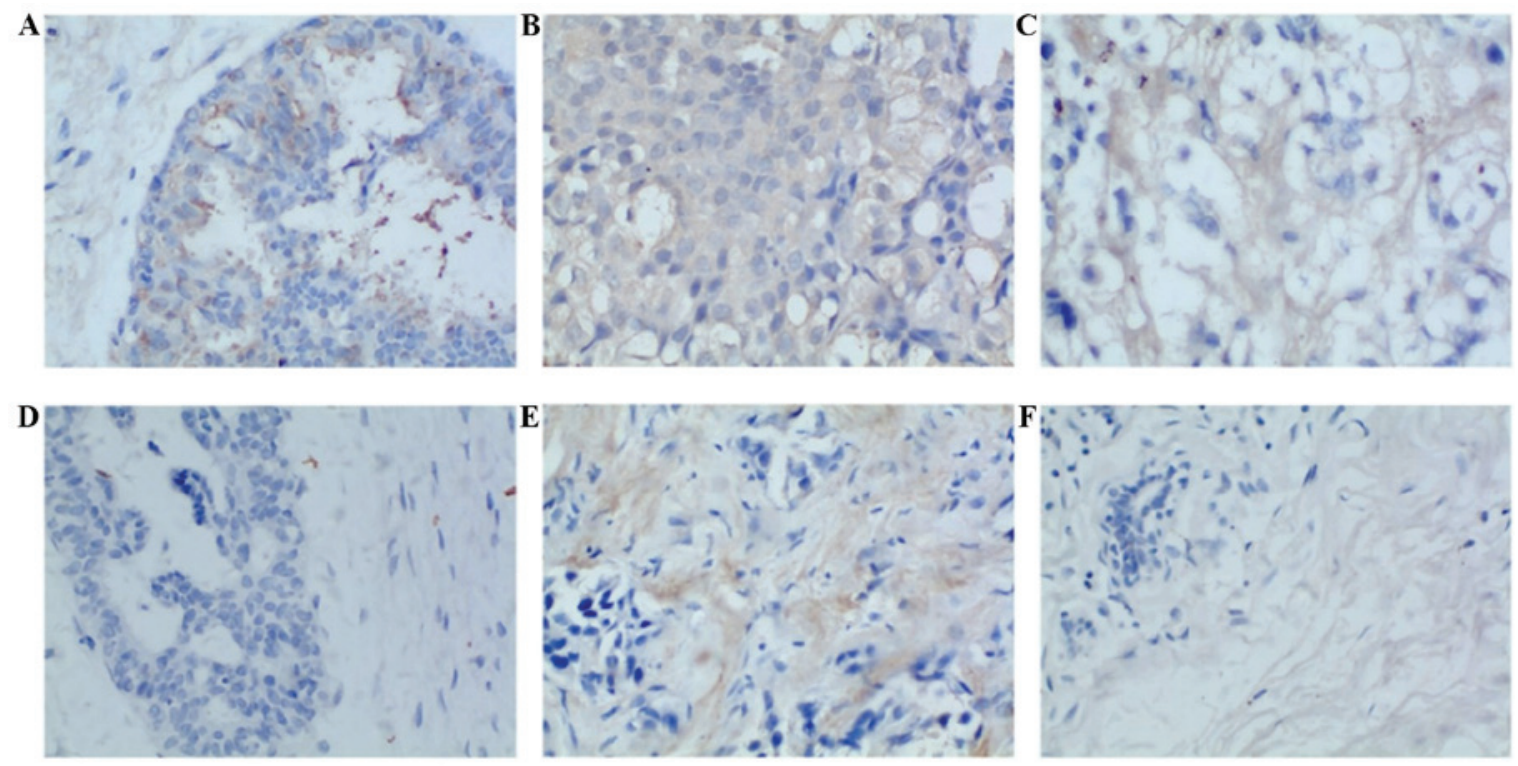

Figure 1. Protein tyrosine phosphatase $\zeta$ (PTPRZ1) and pleiotrophin (PTN) are expressed in human breast cancer, lymph nodes and fibroadenoma tissues (A) Fibroadenoma, PTPRZ1 [SABC method x400, area integral optical density (IOD), 286.87]; (B) breast cancer, PTPRZ1 (SABC method x400, area IOD, 1360.95); (C) lymph nodes, PTPRZ1 (SABC method x400, area IOD, 486.34); (D) fibroadenoma, PTN (SABC method x400, area IOD, 39.77); (E) breast cancer, PTN (SABC method x400, area IOD, 633.78); (F) lymph nodes, PTN (SABC method x400, area IOD, 164.07).

of this subset of TNBC for clinical utility, which has facilitated the assumption that these cancers represent a discrete clinical entity. Clinical case series and reviews have indeed identified a generally poor prognosis for patients with these cancers in comparison with ER-positive and HER2-positive cancers $(1,2,18,19)$. This phenomenon is caused in large part by 
Table III. Association between the expression of PTPRZ1 and PTN and patient clinicopathological features.

\begin{tabular}{|c|c|c|c|c|c|}
\hline \multirow[b]{2}{*}{ Feature } & \multirow[b]{2}{*}{ No. } & \multicolumn{2}{|c|}{ PTN } & \multicolumn{2}{|c|}{ PTPRZ1 } \\
\hline & & Mean \pm SD & P-value & Mean \pm SD & P-value \\
\hline \multicolumn{6}{|l|}{ ER } \\
\hline Positive & 133 & $137.11 \pm 100.33$ & \multirow[t]{2}{*}{0.079} & $364.38 \pm 381.03$ & \multirow[t]{2}{*}{0.337} \\
\hline Negative & 192 & $211.52 \pm 176.14$ & & $464.47 \pm 368.00$ & \\
\hline \multicolumn{6}{|l|}{ PR } \\
\hline Positive & 115 & $147.71 \pm 105.44$ & \multirow[t]{2}{*}{0.240} & $401.84 \pm 403.62$ & \multirow[t]{2}{*}{0.754} \\
\hline Negative & 210 & $199.39 \pm 172.56$ & & $435.56 \pm 361.02$ & \\
\hline \multicolumn{6}{|l|}{ Her-2 } \\
\hline Positive & 78 & $143.72 \pm 47.66$ & \multirow[t]{2}{*}{0.316} & $425.94 \pm 366.50$ & \multirow[t]{2}{*}{0.980} \\
\hline Negative & 247 & $193.09 \pm 172.78$ & & $422.98 \pm 379.66$ & \\
\hline \multicolumn{6}{|l|}{ ALN } \\
\hline Positive & 168 & $195.82 \pm 195.50$ & \multirow[t]{2}{*}{0.472} & $394.61 \pm 313.51$ & \multirow[t]{2}{*}{0.557} \\
\hline Negative & 157 & $165.46 \pm 89.64$ & & $455.02 \pm 432.42$ & \\
\hline \multicolumn{6}{|l|}{ Tumor size } \\
\hline$<2 \mathrm{~cm}$ & 132 & $187.00 \pm 109.29$ & \multirow{3}{*}{0.486} & $464.22 \pm 431.89$ & \multirow[t]{3}{*}{0.568} \\
\hline $2-5 \mathrm{~cm}$ & 157 & $192.75 \pm 194.84$ & & $422.82 \pm 360.06$ & \\
\hline$>5 \mathrm{~cm}$ & 36 & $109.92 \pm 46.38$ & & $278.92 \pm 107.37$ & \\
\hline \multicolumn{6}{|l|}{ Menopause } \\
\hline Yes & 138 & $154.72 \pm 95.08$ & \multirow[t]{2}{*}{0.278} & $431.70 \pm 194.71$ & \multirow[t]{2}{*}{0.893} \\
\hline No & 157 & $200.80 \pm 184.25$ & & $417.75 \pm 362.76$ & \\
\hline
\end{tabular}

PTPRZ1, protein tyrosine phosphatase $\xi$; PTN, pleiotrophin; ER, estrogen receptor; PR, progesterone receptor; ALN, axillary lymph node.

Table IV. Correlation between PTPRZ1 and PTN and the expression of CD24 and CD44.

\begin{tabular}{|c|c|c|c|c|c|c|c|}
\hline & \multirow[b]{2}{*}{ No. } & \multicolumn{2}{|c|}{ PTN } & \multicolumn{2}{|c|}{ CD24 } & \multicolumn{2}{|c|}{ CD44 } \\
\hline & & $\mathrm{CI}$ & P-value & $\mathrm{CI}$ & P-value & CI & P-value \\
\hline PTPRZ1 & 325 & 0.179 & 0.195 & 0.086 & 0.536 & 0.153 & 0.271 \\
\hline
\end{tabular}

PTPRZ1, protein tyrosine phosphatase $\xi$; PTN, pleiotrophin; CI, confidence interval; CD, cluster of differentiation.

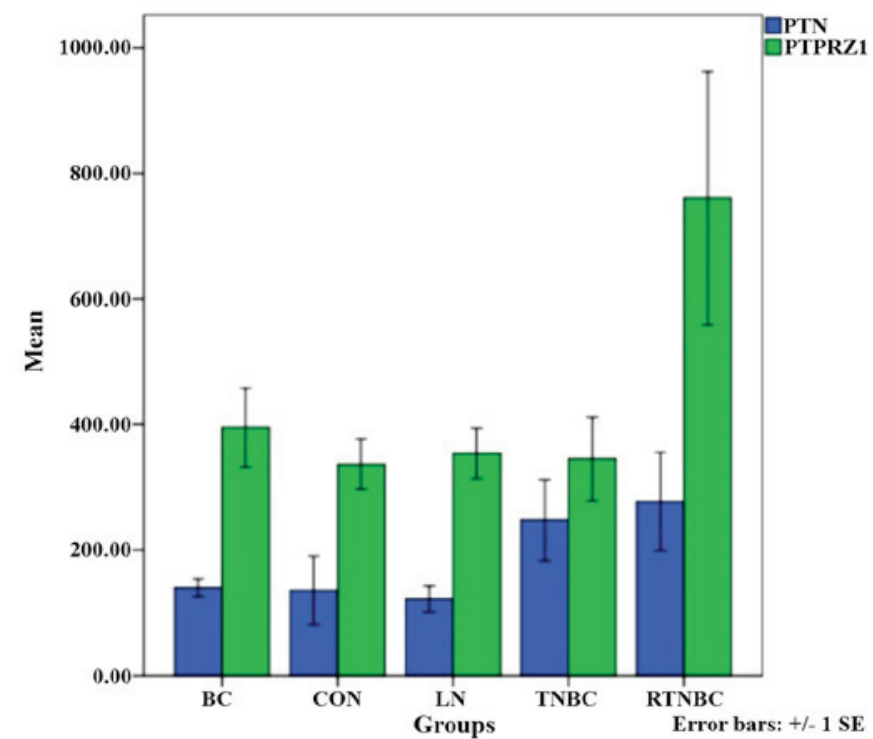

Figure 2. Differential expression of protein tyrosine phosphatase $\zeta$ (PTPRZ1) and pleiotrophin (PTN) in each group. BC, breast cancer; CON, control.

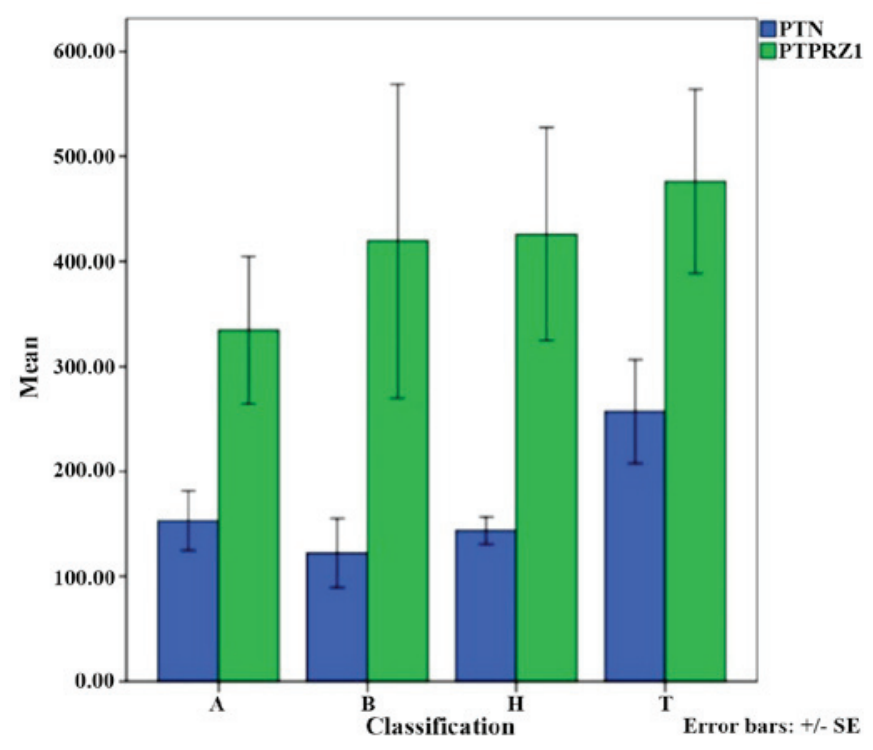

Figure 3. Differential expression of protein tyrosine phosphatase $\zeta$ (PTPRZ1) and pleiotrophin (PTN) in each group. 
the absence of effective targeted therapies with broad activity in breast cancers that are defined only as TNBCs.

Within the TNBC cohort, significant biological diversity exists, which results in the variability of clinical outcomes in response to chemotherapy (19). Lehmann et al (20) identified six defined subtypes within a cohort of TNBC patients, based on gene expression. The basal-like (BL)-1, BL-2, mesenchymal, mesenchymal stem-like (MSL), immunomodulatory, and luminal androgen receptor (LAR) subtypes were identified. Genomic cluster analysis identified specific genes and pathways that characterize these subsets, as follows: proliferative and DNA-repair pathways in the BL1 subtype; epidermal growth factor hepatocyte growth factor receptor and Wnt in the BL2 subtype; immune signaling in the immunomodulatory subtype; cell motility in both the mesenchymal and mesenchymal stem-like subtypes, and hormonal signaling in the luminal androgen receptor subtype. Significant clinical diversity has been observed among these robustly defined molecular subtypes, as evidenced by markedly different responsiveness to neoadjuvant chemotherapy. As an example, $52 \%$ of patients with the BL1 subtype achieve pCR, whereas $0 \%$ of patients with BL2 achieve pCR (21). A meta-analysis of gene expression in 190 cases of TNBC that were identified by IHC and also identified as BL by the intrinsic gene set also revealed considerable clinical diversity in terms of prognosis. Signatures of an activated stroma exhibited a poorer prognosis, whereas for signatures that reflected BRCA1 loss, reduced mTOR signaling, and activation of the Src pathway were associated with a better prognosis.

In conclusion, the present study demonstrated that the expression of PTPRZ1 was significantly higher in the RTNBC group than in the TNBC, BC and control groups. These results indicate that high expression of PTPRZ1 may be an independent risk factor for the recurrence and metastasis of human TNBC. With regard to cancer, previous studies have demonstrated that PTPRZ1 expression is significantly induced by genetic amplification caused by chronic oxidative stress and hypoxic stress through HIF-2 $\alpha(12,22)$. Tumor hypoxia has been demonstrated to play a crucial role in both tumorigenesis and therapeutic resistance. Recently, it has been suggested that hypoxia leads to and maintains the undifferentiated state of tumor stem cells, which contributes to chemoresistance (9,23-26). To study the correlation among PTPRZ1, its ligand PTN, and breast cancer stem cells, the expression of CD24 and CD44 was evaluated in 325 cases of breast cancer by IHC. No significant correlation between the expression of PTPRZ1 and PTN and the expression of CD24 and CD44 was found. Therefore, future studies to further elucidate the mechanism of action of PTPRZ1 are required.

\section{Acknowledgements}

The study was funded by the National Natural Science Foundation of China (grant no. 81441084). The authors thank the American Journal Experts for the language services provided.

\section{References}

1. Lehmann BD and Pietenpol JA: Identification and use of biomarkers in treatment strategies for triple-negative breast cancer subtypes. J Pathol 232: 142-150, 2014.
2. Bosch A, Eroles P, Zaragoza R, Viña JR and Lluch A: Triple-negative breast cancer: Molecular features, pathogenesis, treatment and current lines of research. Cancer Treat Rev 36: 206-215, 2010.

3. Bae YH, Ryu JH, Park HJ, Kim KR, et al: Mutant p53-Notch1 signaling axis is involved in curcumin-induced apoptosis of breast cancer cells. Korean J Physiol Pharmacol 17: 291-297, 2013.

4. Xu Y, Diao L, Chen Y, Liu Y, Wang C, Ouyang T, Li J, Wang T, Fan Z, Fan T, et al: Promoter methylation of BRCA1 in triple-negative breast cancer predicts sensitivity to adjuvant chemotherapy. Ann Oncol 24: 1498-1505, 2013.

5. Hudis CA and Gianni L: Triple-negative breast cancer: An unmet medical need. Oncologist 16 (Suppl 1): 1-11, 2011.

6. Jerónimo C, Costa I, Martins MC, Monteiro P, Lisboa S, Palmeira C, Henrique R, Teixeira MR and Lopes C: Detection of gene promoter hypermethylation in fine needle washings from breast lesions. Clin Cancer Res 9: 3413-3417, 2003.

7. Zhou L, Li K, Luo Y, Tian L, Wang M, Li C and Huang Q: Novel prognostic markers for patients with triple-negative breast cancer. Hum Pathol 44: 2180-2187, 2013.

8. Ismail-Khan R and Bui MM: A review of triple-negative breast cancer. Cancer Control 17: 173-176, 2010.

9. Shitara K, Yamada H, Watanabe K, Shimonaka M and Yamaguchi Y: Brain-specific receptor-type protein-tyrosine phosphatase RPTP beta is a chondroitin sulfate proteoglycan in vivo. J Biol Chem 269: 20189-20193, 1994.

10. Jäger R, List B, Knabbe C, Souttou B, Raulais D, Zeiler T, Wellstein A, Aigner A, Neubauer A and Zugmaier G: Serum levels of the angiogenic factor pleiotrophin in relation to disease stage in lung cancer patients. Br J Cancer 86: 858-863, 2002.

11. Kadomatsu $\mathrm{K}$ and Muramatsu T: Midkine and pleiotrophin in neural development and cancer. Cancer Lett 204: 127-143, 2004.

12. Liu YT, Shang D, Akatsuka S, Ohara H, Dutta KK, Mizushima K, Naito Y, Yoshikawa T, Izumiya M, Abe K, et al: Chronic oxidative stress causes amplification and overexpression of ptprz1 protein tyrosine phosphatase to activate beta-catenin pathway. Am J Pathol 171: 1978-1988, 2007.

13. Tamura H, Fukada M, Fujikawa A and Noda M: Protein tyrosine phosphatasereceptortypeZisinvolved inhippocampus-dependent memory formation through dephosphorylation at Y1105 on p190 RhoGAP. Neurosci Lett 399: 33-38, 2006.

14. Pariser H, Perez-Pinera P, Ezquerra L, Herradon G and Deuel TF: Pleiotrophin stimulates tyrosine phosphorylation of beta-adducin through inactivation of the transmembrane receptor protein tyrosine phosphatase beta/zeta. Biochem Biophys Res Commun 335: 232-239, 2005

15. Pariser H, Ezquerra L, Herradon G, Perez-Pinera P and Deuel TF: Fyn is a downstream target of the pleiotrophin/receptor protein tyrosine phosphatase beta/zeta-signaling pathway: Regulation of tyrosine phosphorylation of Fyn by pleiotrophin. Biochem Biophys Res Commun 332: 664-669, 2005.

16. Rokavec M, Wu W and Luo JL: IL6-mediated suppression of miR-200c directs constitutive activation of an inflammatory signaling circuit that drives transformation and tumorigenesis. Mol Cell 45: 777-789, 2012.

17. Yang $\mathrm{F}$ and $\mathrm{Li} \mathrm{J}$ : WHO classification of tumors of the breast. Zhonghua Wai Ke Za Zhi 52: 1-3, 2014.

18. Haffty BG, Yang Q, Reiss M, Kearney T, Higgins SA, Weidhaas J, Harris L, Hait W and Toppmeyer D: Locoregional relapse and distant metastasis in conservatively managed triple negative early-stage breast cancer. J Clin Oncol 24: 5652-5657, 2006.

19. Guarneri V, Dieci MV and Conte P: Relapsed triple-negative breast cancer: Challenges and treatment strategies. Drugs 73: 1257-1265, 2013.

20. Lehmann BD, Bauer JA, Chen X, Sanders ME, Chakravarthy AB, Shyr Y and Pietenpol JA: Identification of human triple-negative breast cancer subtypes and preclinical models for selection of targeted therapies. J Clin Invest 121: 2750-2767, 2011.

21. MasudaH,BaggerlyKA,Wang Y,Zhang Y,Gonzalez-Angulo AM, Meric-Bernstam F, Valero V, Lehmann BD, Pietenpol JA, Hortobagyi GN, et al: Differential response to neoadjuvant chemotherapy among 7 triple-negative breast cancer molecular subtypes. Clin Cancer Res 19: 5533-5540, 2013.

22. Wang V, Davis DA, Veeranna RP, Haque M and Yarchoan R: Characterization of the activation of protein tyrosine phosphatase, receptor-type, Z polypeptide 1 (PTPRZ1) by hypoxia inducible factor-2 alpha. PLoS One 5: e9641, 2010. 
23. Meng K, Rodriguez-Peña A, Dimitrov T, Chen W, Yamin M, Noda M and Deuel TF: Pleiotrophin signals increased tyrosine phosphorylation of beta beta-catenin through inactivation of the intrinsic catalytic activity of the receptor-type protein tyrosine phosphatase beta/zeta. Proc Natl Acad Sci USA 97: 2603-2608, 2000.

24. Ulbricht U1, Eckerich C, Fillbrandt R, Westphal M and Lamszus K: RNA interference targeting protein tyrosine phosphatase beta suppresses glioblastoma growth in vitro and in vivo. J Neurochem 98: 1497-1506, 2006.
25. Foehr ED, Lorente G, Kuo J, Ram R, Nikolich K and Urfer R: Targeting of the receptor protein tyrosine phosphatase beta with a monoclonal antibody delays tumor growth in a glioblastoma model. Cancer Res 66: 2271-2278, 2006.

26. Feng ZJ, Gao SB, Wu Y, Xu XF, Hua X and Jin GH: Lung cancer cell migration is regulated via repressing growth factor PTN/RPTP $\beta / \zeta$ signaling by menin. Oncogene 29: 5416-5426, 2010. 